\title{
New Quasi-Coincidence Point Polynomial Problems
}

\author{
Yi-Chou Chen ${ }^{1}$ and Hang-Chin Lai' \\ ${ }^{1}$ Department of General Education, National Army Academy, Taoyuan 320, Taiwan \\ ${ }^{2}$ Department of Mathematics, National Tsing Hua University, Hsinchu 300, Taiwan
}

Correspondence should be addressed to Yi-Chou Chen; cycu.chou@gmail.com

Received 30 April 2013; Accepted 10 July 2013

Academic Editor: Tamaki Tanaka

Copyright (C) 2013 Y.-C. Chen and H.-C. Lai. This is an open access article distributed under the Creative Commons Attribution License, which permits unrestricted use, distribution, and reproduction in any medium, provided the original work is properly cited.

Let $F: \mathbb{R} \times \mathbb{R} \rightarrow \mathbb{R}$ be a real-valued polynomial function of the form $F(x, y)=a_{s}(x) y^{s}+a_{s-1}(x) y^{s-1}+\cdots+a_{0}(x)$, where the degree $s$ of $y$ in $F(x, y)$ is greater than or equal to 1 . For arbitrary polynomial function $f(x) \in \mathbb{R}[x], x \in \mathbb{R}$, we will find a polynomial solution $y(x) \in \mathbb{R}[x]$ to satisfy the following equation: $(*): F(x, y(x))=a f(x)$, where $a \in \mathbb{R}$ is a constant depending on the solution $y(x)$, namely, a quasi-coincidence (point) solution of $(*)$, and $a$ is called a quasi-coincidence value. In this paper, we prove that (i) the leading coefficient $a_{s}(x)$ must be a factor of $f(x)$, and (ii) each solution of $(*)$ is of the form $y(x)=-a_{s-1}(x) / s a_{s}(x)+\lambda p(x)$, where $\lambda$ is arbitrary and $p(x)=c\left(f(x) / a_{s}(x)\right)^{1 / s}$ is also a factor of $f(x)$, for some constant $c \in \mathbb{R}$, provided the equation $(*)$ has infinitely many quasi-coincidence (point) solutions.

\section{Introduction and Preliminaries}

Let $F: \mathbb{Q}(\alpha) \times \mathbb{Q}(\alpha) \rightarrow \mathbb{Q}(\alpha)$ (where $\alpha$ is an algebraic number) be a polynomial function. Lenstra [1] investigated that $F(x, y(x))=0$. He solved a polynomial function $y=$ $y(x) \in \mathbb{Q}(\alpha)[x]$ and derived to find a polynomial $y=y(x)$ satisfying an $x \in \mathbb{Q}(\alpha)[x]$ as a fixed point of the polynomial equation. That is,

$$
F(x, y(x))=x
$$

has a polynomial solution $y(x) \in \mathbb{Q}(\alpha)[x]$.

Further, Tung $[2,3]$ extended (1) to solve $y(x) \in \mathbb{K}[x](\mathbb{K}$ is a field) for the following equation:

$$
F(x, y(x))=a x^{m}
$$

where $a \in \mathbb{K}$ is a constant depending on the polynomial solution $y(x)$ and $m \in \mathbb{N}$ a given positive integer.

Recently, Lai and Chen $[4,5]$ extended (2) to solve $y(x) \in$ $\mathbb{R}[x]$ to satisfy the polynomial equation as the form:

$$
F(x, y(x))=a p^{m}(x), \quad x \in \mathbb{R},
$$

where $a \in \mathbb{R}, p(\cdot)$ is an irreducible polynomial in $x \in \mathbb{R}$ and the polynomial function $F(x, y): \mathbb{R} \times \mathbb{R} \rightarrow \mathbb{R}$ is written by

$$
F(x, y)=\sum_{i=0}^{s} a_{i}(x) y^{i} \quad \text { with } s \geq 1,
$$

where $s=\operatorname{deg}_{y} F$ denotes the degree of $y$ in $F(x, y)$.

Definition 1 (see [4]). A polynomial function $y=y(x)$ satisfying (3) is called a quasi-fixed solution corresponding to some real number $a$. This number $a$ is called a quasi-fixed value corresponding to the polynomial solutions $y=y(x)$.

In mathematics, a coincidence point (or simply coincidence) of two mappings is a point in their domain having the same image point under both mappings. Coincidence theory (the study of coincidence points) is, in most settings, a generalization of fixed point theory.

In this paper, we define a more general coincidence (point) problem in which the $f(x) \in \mathbb{R}[x]$ is replaced by the irreducible polynomial power $p^{m}(x) \in \mathbb{R}[x]$ throughout this 
paper, where $f(x)$ is an arbitrary polynomial. Then, we restate (3) as the following equation:

$$
F(x, y)=a f(x) .
$$

It is a new development coincidence point-like problem. We call the polynomial solution $y=y(x)$ for (5) as a quasicoincidence (point) solution. Precisely, we give the following definition like Definition 1.

Definition 2. A polynomial function $y=y(x)$ satisfying (5) is called a quasi-coincidence (point) solution corresponding to some real number $a$. This number $a$ is called a quasicoincidence value corresponding to the polynomial solutions $y=y(x)$.

The number of all solutions in (5) may be infinitely many, or finitely many, or not solvable.

Since there may have many solutions corresponding to the number $a$, for convenience, we use the following notations to represent different situations:

(1) $\mathrm{Qcs}_{F}$, the set of all quasi-coincidence solutions satisfying (5),

(2) $\mathrm{Qcv}_{F}$, the set of all quasi-coincidence values satisfying (5),

(3) $\operatorname{Qcs}_{F}(a)$, the set of all quasi-coincidence solutions $y(x)$ corresponding to a fixed quasi-coincidence value $a$.

Evidently,

$$
\begin{gathered}
\operatorname{Qcs}_{F}=\bigcup_{a \in \operatorname{Qcv}_{F}} \operatorname{Qcs}_{F}(a), \\
\operatorname{Qcs}_{F}(a) \bigcap \operatorname{Qcs}_{F}(b)=\emptyset
\end{gathered}
$$

for any $a \neq b$ in $\mathrm{Qcv}_{F}$. Moreover, for each $a \in \mathbb{R}$, the cardinal number of $\mathrm{Qcs}_{F}(a)$, denoted by $\left|\mathrm{Q} \mathrm{cs}_{F}(a)\right|$, satisfies the following condition:

$$
\left|\operatorname{Qcs}_{F}(a)\right| \leq \operatorname{deg}_{y} F(x, y) .
$$

In Section 2, we derive some properties of quasicoincidence solutions of $F(x, y)$. If (5) has infinitely many quasi-coincidence solutions, the concerned properties are described in Section Section 3.

Throughout the paper, we denote the polynomial function by

$$
\begin{aligned}
F(x, y)= & a_{s}(x) y^{s}+a_{s-1}(x) y^{s-1} \\
& +\cdots+a_{1}(x) y+a_{0}(x) \\
= & \sum_{i=0}^{s} a_{i}(x) y^{i} .
\end{aligned}
$$

\section{Auxiliary Lemmas}

For convenience, we explain some interesting properties of quasi-coincidence point solutions as the following lemmas. Throughout this paper, we consider (5) for polynomial function (9) and arbitrary polynomial $f(x)$ in $\mathbb{R}[x]$.
Lemma 3. Let $y_{1}(x) \in Q c s_{F}(a), y_{2}(x) \in Q c s_{F}(b), a \neq b$ in $Q c v_{F}$. Then,

$$
y_{1}(x)-y_{2}(x)=d p(x) \quad \text { for some } d \in \mathbb{R},
$$

and this $p(x)$ is divisible $f(x)$; that is, $p(x) \mid f(x)$.

Proof. Since $y_{1}(x) \neq y_{2}(x)$ in $\operatorname{Qcs}_{F}$ correspond to $a \neq b$ in $\mathrm{QCv}_{F}$, respectively, thus

$$
\begin{aligned}
& F\left(x, y_{1}(x)\right)=a f(x), \\
& F\left(x, y_{2}(x)\right)=b f(x) .
\end{aligned}
$$

Subtracting the above two equations and using binomial formula, it yields

$$
\begin{aligned}
(a-b) f(x)= & F\left(x, y_{1}(x)\right)-F\left(x, y_{2}(x)\right) \\
= & a_{s}(x)\left[y_{1}^{s}(x)-y_{2}^{s}(x)\right]+a_{s-1}(x) \\
& \times\left[y_{1}^{s-1}(x)-y_{2}^{s-1}(x)\right] \\
& +\cdots+a_{1}(x)\left[y_{1}(x)-y_{2}(x)\right] \\
= & {\left[y_{1}(x)-y_{2}(x)\right] } \\
& \times\left[a_{s}(x) G_{s}\left(y_{1}(x), y_{2}(x)\right)\right. \\
& \quad+a_{s-1}(x) G_{s-1}\left(y_{1}(x), y_{2}(x)\right) \\
& \left.\quad+\cdots+a_{1}(x)\right] \\
= & {\left[y_{1}(x)-y_{2}(x)\right] Q\left(x, y_{1}(x), y_{2}(x)\right), }
\end{aligned}
$$

where $G_{j}\left(y_{1}(x), y_{2}(x)\right)=y_{1}^{j-1}(x)+y_{1}^{j-2}(x) y_{2}(x)+\cdots+$ $y_{2}^{j-1}(x)$, for $j=s, s-1, \ldots, 2,1$. Evidently, the factor $y_{1}(x)-$ $y_{2}(x)$ is divisible to the term $(a-b) f(x)$.

Since $a \neq b$,

$$
y_{1}(x)-y_{2}(x)=d p(x)
$$

for a real number $d \in \mathbb{R}$ and some factor $p(x)$ of $f(x)$.

In Lemma 3, the difference of any two distinct quasicoincidence solutions corresponding to distinct values is a factor of $f(x)$. Thus, we define a class of this factor as follows.

Notation. (i) Let $p(x)$ be a factor of $f(x)$, and we denote $\Phi(p(x))=\{\alpha p(x): \alpha \in \mathbb{R}\}$.

(ii) Let $y(x)$ be an arbitrary polynomial in $\mathbb{R}[x]$, and we denote $y(x)+\Phi(p(x))=\{y(x)+\alpha p(x): \alpha \in \mathbb{R}\}$.

It is obvious that for any $y(x), p(x) \in \mathbb{R}[x]$, then the cardinal number

$$
|y(x)+\Phi(p(x))|=\infty
$$

For convenience, we explain the relations of $\mathrm{Qcs}_{F}$ and $\Phi(p(x))$ in the following lemma. 
Lemma 4. Let $y(x) \in \operatorname{Qcs}_{F}(a)$ for some $a \in \mathbb{R}$, then

$$
\begin{aligned}
\operatorname{Qcs}_{F}= & Q c s_{F}(a) \\
& \bigcup\left(\bigcup_{p(x) \mid f(x)}\left\{(y(x)+\Phi(p(x))) \cap Q c s_{F}\right\}\right) .
\end{aligned}
$$

Proof. For any $y_{1}(x) \in \mathrm{Qcs}_{F} \backslash \mathrm{Q} \operatorname{cs}_{F}(a)$, then $y_{1}(x) \in \mathrm{Qcs}(b)$ for some $b \in \mathrm{Qcv}_{F}$. By Lemma 3, we have

$$
y_{1}(x)-y(x) \in \Phi(p(x))
$$

for some factor $p(x)$ of $f(x)$. Then,

$$
y_{1}(x) \in \bigcup_{p(x) \mid f(x)}\{y(x)+\Phi(p(x))\} .
$$

That is,

$$
\operatorname{Qcs}_{F} \subseteq \operatorname{Qcs}_{F}(a) \bigcup\left(\bigcup_{p(x) \mid f(x)}\{y(x)+\Phi(p(x))\}\right) .
$$

Moreover, by (6), $\operatorname{Qcs}_{F}(a) \subseteq \mathrm{Qcs}_{F}$, then it follows that

$$
\begin{aligned}
\operatorname{Qcs}_{F}= & \operatorname{Qcs}_{F}(a) \\
& \bigcup\left(\bigcup_{p(x) \mid f(x)}\left\{(y(x)+\Phi(p(x))) \cap \mathrm{Qcs}_{F}\right\}\right) .
\end{aligned}
$$

We will use the definitions of "the pigeonhole principle;" it could concert to Grimaldi [6], and the relation can be explained as the following lemma.

Lemma 5. Suppose that the cardinal number $\left|Q c s_{F}\right|=\infty$. For any $y(x) \in Q c s_{F}$, there exists a factor $p(x)$ of $f(x)$ such that the cardinal number

$$
\left|(y(x)+\Phi(p(x))) \cap Q c s_{F}\right|=\infty .
$$

Proof. Let $y(x) \in \mathrm{Qcs}_{F}$, then $y(x) \in \operatorname{Qcs}_{F}(a)$ for some $a \in \mathbb{R}$. Since $\left|\mathrm{Q} c s_{F}\right|=\infty$ and $|\mathrm{Qcs} F(a)| \leq s$, by Lemma 4 , we obtain

$$
\left|\bigcup_{p(x) \mid f(x)}\left(\{y(x)+\Phi(p(x))\} \cap \mathrm{Qcs}_{F}\right)\right|=\infty,
$$

it yields

$$
\sum_{p(x) \mid f(x)}\left|(y(x)+\Phi(p(x))) \cap \mathrm{Qcs}_{F}\right|=\infty .
$$

Moreover, the number of all factor $p(x)$ of $f(x)$ is at most $2^{\operatorname{deg} f(x)}$, by pigeonhole's principle, it leads to

$$
\left|(y(x)+\Phi(p(x))) \cap Q \operatorname{Qss}_{F}\right|=\infty
$$

for some factor $p(x)$ of $f(x)$.

In order to know if the intersection of two sets still has infinite solutions, we state the following result to give an explanation.
Lemma 6. Suppose that the cardinal number $\left|Q c s_{F}\right|=\infty$, for any $y_{1}(x) \neq y_{2}(x) \in Q c s_{F}$, there exist some factors $p_{1}(x)$ and $p_{2}(x)$ of $f(x)$ such that

$$
\left|\left(y_{1}(x)+\Phi\left(p_{1}(x)\right)\right) \cap\left(y_{2}(x)+\Phi\left(p_{2}(x)\right)\right) \cap Q c s_{F}\right|=\infty .
$$

Proof. Let $y_{1}(x) \in \mathrm{Qcs}_{F}$ and since $\left|\mathrm{Q} \mathrm{cs}_{F}\right|=\infty$, by Lemma 5, there exists a factor $p_{1}(x)$ of $f(x)$ such that

$$
\left|\left(y_{1}(x)+\Phi\left(p_{1}(x)\right)\right) \cap \mathrm{Q} \operatorname{cs}_{F}\right|=\infty .
$$

Moreover, $y_{2}(x) \in \mathrm{Qcs}_{F}$, by Lemma 4,

$$
\operatorname{Qcs}_{F} \subseteq \operatorname{Qcs}_{F}(b) \bigcup\left(\bigcup_{p_{2}(x) \mid f(x)}\left\{y_{2}(x)+\Phi\left(p_{2}(x)\right)\right\}\right)
$$

for some constant $b \in \mathbb{R}$ and some factor $p_{2}(x)$ of $f(x)$. Thus,

$$
\begin{aligned}
& \left(\left\{y_{1}(x)+\Phi\left(p_{1}(x)\right)\right\} \cap \mathrm{Qcs}_{F}\right) \\
& \quad \subseteq \mathrm{Qcs}_{F} \subseteq \mathrm{Qcs}_{F}(b) \bigcup\left(\bigcup_{p_{2}(x) \mid f(x)}\left\{y_{2}(x)+\Phi\left(p_{2}(x)\right)\right\}\right) .
\end{aligned}
$$

Since $\left|\mathrm{Qcs}_{F}(b)\right| \leq s$ and the number of all factor to $f(x)$ is at most $2^{\operatorname{deg} f(x)}$, by pigeonhole's principle and (25), we have

$$
\begin{aligned}
& \left|\left(y_{2}(x)+\Phi\left(p_{2}(x)\right)\right) \cap Q \operatorname{cs}_{F}\right|=\infty, \\
& \mid\left(y_{1}(x)+\Phi\left(p_{1}(x)\right)\right) \\
& \cap\left(y_{2}(x)+\Phi\left(p_{2}(x)\right)\right) \cap Q \operatorname{cs}_{F} \mid=\infty
\end{aligned}
$$

for some factor $p_{2}(x)$ of $f(x)$.

Up to now, we have not shown that the factor $p(x)$ is uniquely existed. Eventually, if the number of all solutions is infinitely many, then the factor $p(x)$ of $f(x)$ is unique up to the choice of the solution $y(x)$.

Lemma 7. Assume that the cardinal number $\left|Q c s_{F}\right|=\infty$, then for any $y_{1}(x), y_{2}(x) \in Q c s_{F}$, one has

$$
y_{1}(x)-y_{2}(x)=\lambda p(x)
$$

for some constant $\lambda \in \mathbb{R}$ and some factor $p(x)$ of $f(x)$ (this $p(x)$ is independent to the choice of $y_{1}(x)$ and $\left.y_{2}(x)\right)$.

Proof. Let $y_{1}(x) \neq y_{2}(x) \in \mathrm{Qcs}_{F}$, by Lemma 6, we have

$$
\begin{aligned}
& \mid\left(y_{1}(x)+\Phi\left(p_{1}(x)\right)\right) \\
& \cap\left(y_{2}(x)+\Phi\left(p_{2}(x)\right)\right) \cap \mathrm{Qcs}_{F} \mid=\infty
\end{aligned}
$$

for some factors $p_{1}(x), p_{2}(x)$ of $f(x)$.

Let $g_{1}(x) \neq g_{2}(x) \in\left(y_{1}(x)+\Phi\left(p_{1}(x)\right)\right) \cap\left(y_{2}(x)+\right.$ $\left.\Phi\left(p_{2}(x)\right)\right) \cap \mathrm{Qcs}_{F}-\left\{y_{1}(x), y_{2}(x)\right\}$, then

$$
\begin{aligned}
& g_{1}(x) \in y_{1}(x)+\Phi\left(p_{1}(x)\right), \\
& g_{2}(x) \in y_{1}(x)+\Phi\left(p_{1}(x)\right), \\
& g_{1}(x) \in y_{2}(x)+\Phi\left(p_{2}(x)\right), \\
& g_{2}(x) \in y_{2}(x)+\Phi\left(p_{2}(x)\right) .
\end{aligned}
$$


By Lemma 6, it yields

$$
\begin{aligned}
& g_{1}(x)-y_{1}(x)=\lambda_{1} p_{1}(x), \\
& g_{2}(x)-y_{1}(x)=\lambda_{2} p_{1}(x), \\
& g_{1}(x)-y_{2}(x)=\lambda_{3} p_{2}(x), \\
& g_{2}(x)-y_{2}(x)=\lambda_{4} p_{2}(x)
\end{aligned}
$$

for some constants $\lambda_{1}, \lambda_{2}, \lambda_{3}$, and $\lambda_{4} \in \mathbb{R}$, and consequently

$$
\begin{aligned}
g_{2}(x)-g_{1}(x) & =\left(g_{2}(x)-y_{1}(x)\right)-\left(g_{1}(x)-y_{1}(x)\right) \\
& =\left(\lambda_{2}-\lambda_{1}\right) p_{1}(x), \\
g_{2}(x)-g_{1}(x)= & \left(g_{2}(x)-y_{2}(x)\right) \\
& -\left(g_{1}(x)-y_{2}(x)\right)=\left(\lambda_{4}-\lambda_{3}\right) p_{2}(x) .
\end{aligned}
$$

This implies that

$$
\left(\lambda_{2}-\lambda_{1}\right) p_{1}(x)=\left(\lambda_{4}-\lambda_{3}\right) p_{2}(x)
$$

and $p_{1}(x)=p_{2}(x)$. Therefore,

$$
\begin{aligned}
y_{1}(x)-y_{2}(x) & =\left(g_{1}(x)-y_{2}(x)\right)-\left(g_{1}(x)-y_{1}(x)\right) \\
& =\left(\lambda_{3}-\lambda_{1}\right) p_{1}(x) .
\end{aligned}
$$

Consequently, the factor $p(x)$ is uniquely existed.

By the above preparations, at first we consider the polynomial function $F(x, y)$ with $\operatorname{deg}_{y} F=1$ as the form

$$
F(x, y)=a_{1}(x) y+a_{0}(x)
$$

Then, we consider the theorem of problem as

$$
F(x, y)=a f(x)
$$

In the following theorem, we integrate the above type as follows.

Theorem 8. Let $F(x, y)$ be a polynomial function with $\operatorname{deg}_{y} F=1$ as the form $F(x, y)=a_{1}(x) y+a_{0}(x)=a f(x)$ for some $a \in \mathbb{R}$ (where polynomial function $f(x)$ is given). If the cardinal number $\left|Q c v_{F}\right| \geq 2\left(=\operatorname{deg}_{y} F+1\right)$, then

(i) $a_{1}(x)$ is some factor of $f(x)$,

(ii) any solution of (5) is of the form:

$$
y(x)=-\frac{a_{0}(x)}{a_{1}(x)}+\lambda p(x)
$$

for some $\lambda \in \mathbb{R}$ and some factor $p(x)$ of $f(x)$,

(iii) the cardinal number $\left|Q c s_{F}\right|=\infty$.
Proof. Since $\left|\mathrm{Qcv}_{F}\right| \geq 2$, we see that there are two distinct quasi-fixed values $a, b \in \mathrm{QCv}_{F}$ corresponding to two distinct solutions $y_{1}(x), y_{2}(x)$ in $\mathrm{Q} \mathrm{cs}_{F}$ such that

$$
\begin{aligned}
& F\left(x, y_{1}(x)\right)=a f(x), \\
& F\left(x, y_{2}(x)\right)=b f(x) .
\end{aligned}
$$

(i) It follows that

$$
\begin{aligned}
& F\left(x, y_{1}(x)\right)=a_{1}(x) y_{1}(x)+a_{0}(x)=a f(x), \\
& F\left(x, y_{2}(x)\right)=a_{1}(x) y_{2}(x)+a_{0}(x)=b f(x) .
\end{aligned}
$$

By (40)-(41), we get

$$
a_{1}(x)\left(y_{1}(x)-y_{2}(x)\right)=(a-b) f(x) .
$$

It follows that $a_{1}(x)$ must be a factor of $f(x)$ and

$$
y_{1}(x)-y_{2}(x)=\frac{(a-b) f(x)}{a_{1}(x)} \in \mathbb{R}[x]
$$

(ii) By (40), we have

$$
\begin{aligned}
a_{0}(x) & =a f(x)-a_{1}(x) y_{1}(x) \\
& =a_{1}(x)\left(a \frac{f(x)}{a_{1}(x)}-y_{1}(x)\right) .
\end{aligned}
$$

Thus, (i) and (45) imply $a_{1}(x) \mid a_{0}(x)$, and by (44), $y_{1}(x)$ can be written as

$$
y_{1}(x)=\frac{a f(x)-a_{0}(x)}{a_{1}(x)} .
$$

Moreover, we derive

$$
\begin{aligned}
F(x, y) & =a_{1}(x) y+a_{0}(x) \\
& =a_{1}(x)\left(y-y_{1}(x)\right)+\left(a_{1}(x) y_{1}(x)+a_{0}(x)\right)
\end{aligned}
$$

by $(40)=a_{1}(x)\left(y-y_{1}(x)\right)+a f(x)$.

For any $y(x) \in \mathrm{Qcs}_{F}$, we have

$$
F(x, y(x))=\tilde{a} f(x)
$$

for some $\tilde{a} \in \mathbb{R}$. By (47) and (48), it follows that

$$
a_{1}(x)\left(y(x)-y_{1}(x)\right)+a f(x)=\tilde{a} f(x)
$$

Hence,

$$
y(x)-y_{1}(x)=\frac{(\tilde{a}-a) f(x)}{a_{1}(x)} .
$$


Then,

$$
\begin{aligned}
y(x) & =y_{1}(x)+\frac{(\tilde{a}-a) f(x)}{a_{1}(x)} \\
\text { by }(46) & =\frac{a f(x)-a_{0}(x)}{a_{1}(x)}+\frac{(\tilde{a}-a) f(x)}{a_{1}(x)} \\
& =\frac{-a_{0}(x)}{a_{1}(x)}+\frac{\tilde{a} f(x)}{a_{1}(x)} .
\end{aligned}
$$

Therefore,

$$
y(x)=\frac{-a_{0}(x)}{a_{1}(x)}+\lambda p(x) \text { for some factor } p(x) \text { of } f(x) .
$$

(Note that this $p(x)=f(x) / a_{1}(x)$ is only dependent on the choice of $F(x, y)$ and $f(x)$.)

(iii) Actually in (ii), for any $\lambda \in \mathbb{R}, y(x)=-a_{0}(x) / a_{1}(x)+$ $\lambda f(x) / a_{1}(x)$ is also a quasi-coincidence solution for $F(x, y)$. The reason is

$$
\begin{aligned}
F(x, y(x)) & =a_{1}(x) y(x)+a_{0}(x) \\
& =a_{1}(x)\left(\frac{-a_{0}(x)}{a_{1}(x)}+\lambda \frac{f(x)}{a_{1}(x)}\right)+a_{0}(x) \\
& =\lambda f(x) .
\end{aligned}
$$

This shows that $(*)$ has infinitely many solutions (i.e., $\left.\left|\mathrm{Q} \mathrm{cs}_{F}\right|=\infty\right)$.

Remark 9. Notice that in the case of $\operatorname{deg}_{y} F=1$ and $\left|Q \mathrm{cs}_{F}\right|<\infty$, the number of all quasi-coincidence values cannot be larger than 1 . Otherwise, it will contract the result of Theorem 8; the case (iii) means that " $\left|\mathrm{Q} \mathrm{cv}_{F}\right| \geq 2$, and then $\left|\mathrm{Q} \operatorname{cs}_{F}\right|=\infty "$.

\section{Main Theorems}

In this section, we consider (5) for polynomial function $F(x, y)$ in (9); that is,

$$
F(x, y)=\sum_{i=0}^{s} a_{i}(x) y^{i} \quad \text { with } s \geq 2 .
$$

A given polynomial function $f(x)$ in $\mathbb{R}[x]$ and $F(x, y)$ has at least $s+1$ distinct quasi-coincidence solutions satisfying some conditions, that is, $y_{1}(x), y_{2}(x), y_{3}(x), \ldots, y_{s+1}(x), \ldots$ According to the above assumptions, we could derive the following theorem.

Theorem 10. Suppose that the cardinal number $\left|Q c s_{F}\right|=\infty$ and for each $y(x) \in Q c s_{F}$ can be represented as the form

$$
y(x)=y_{1}(x)+\lambda p(x), \quad \lambda \in \mathbb{R}
$$

for some $y_{1}(x), p(x) \in \mathbb{R}[x]$. Then, $p^{s}(x) \mid f(x)$, and so the polynomial $F(x, y)$ can be represented as

$$
F(x, y)=\sum_{i=0}^{s} c_{i} \frac{f(x)}{p^{i}(x)}\left(y-y_{1}(x)\right)^{i}
$$

for constants $c_{i} \in \mathbb{R}$.
Proof. Let $y_{i}(x)$ be distinct quasi-coincidence solutions of $F(x, y)$ corresponding to quasi-coincidence values $a_{i}, 1 \leq i \leq$ $s+1$ such that

$$
F\left(x, y_{i}(x)\right)=a_{i} f(x)
$$

Choose $i=1, F\left(x, y_{1}(x)\right)=a_{1} f(x)$. When $y-y_{1}(x)$ divides the function $F(x, y)$, we get

$$
F(x, y)=\left(y-y_{1}(x)\right) F_{1}(x, y)+a_{1} f(x),
$$

where $F_{1}(x, y)$ is the quotient and $a_{1} f(x)$ is the remainder. From the above identity, taking $y=y_{2}(x)$, it becomes

$$
\begin{aligned}
F\left(x, y_{2}(x)\right) & =\left(y_{2}(x)-y_{1}(x)\right) F_{1}\left(x, y_{2}(x)\right)+a_{1} f(x) \\
& =a_{2} f(x) .
\end{aligned}
$$

Then,

$$
\left(y_{2}(x)-y_{1}(x)\right) F_{1}\left(x, y_{2}(x)\right)=\left(a_{2}-a_{1}\right) f(x) .
$$

By (55), $y_{2}(x)-y_{1}(x)=\lambda_{2} p(x)$, it yields

$$
\begin{aligned}
F_{1}\left(x, y_{2}(x)\right) & =\left(\frac{\left(a_{2}-a_{1}\right)}{\lambda_{2}}\right) \frac{f(x)}{p(x)} \\
& =d_{2} \frac{f(x)}{p(x)} \in \mathbb{R}[x] \quad \text { for } d_{2}=\frac{\left(a_{2}-a_{1}\right)}{\lambda_{2}} .
\end{aligned}
$$

Hence,

$$
F_{1}(x, y)=\left(y-y_{2}(x)\right) F_{2}(x, y)+d_{2} \frac{f(x)}{p(x)}
$$

Continuing this process from $i=2$ to $s-1$, we obtain

$$
F_{i}(x, y)=\left(y-y_{i+1}(x)\right) F_{i+1}(x, y)+d_{i+1} \frac{f(x)}{p^{i}(x)}
$$

for some $d_{i+1} \in \mathbb{R}, i=1,2, \ldots, s-1$. Finally, we could get

$$
F_{s-1}(x, y)=\left(y-y_{s}(x)\right) F_{s}(x)+d_{s} \frac{f(x)}{p^{s-1}(x)}
$$

$F_{s}(x)$ does not contain the variable $y$ since $\operatorname{deg}_{y} F=s$. By the assumption (57), $F\left(x, y_{s+1}(x)\right)=a_{s+1} f(x)$. It follows that

$$
F_{s}(x)=\lambda \frac{f(x)}{p^{s}(x)} \in \mathbb{R}[x] \quad \text { for some constant } \lambda \in \mathbb{R} .
$$


Consequently,

$$
\begin{aligned}
& F(x, y)=\left(y-y_{1}(x)\right) F_{1}(x, y)+a_{1} f(x) \\
& =\left(y-y_{1}(x)\right) \\
& \times\left(\left(y-y_{2}(x)\right) F_{2}(x, y)+d_{2} \frac{f(x)}{p(x)}\right)+a_{1} f(x) \\
& =\ldots \\
& =\left(y-y_{1}(x)\right)\left(\left(y-y_{2}(x)\right)\right. \\
& \times\left(\cdots \left(\left(y-y_{s}(x)\right) F_{s}(x)\right.\right. \\
& \left.\left.+d_{s} \frac{f(x)}{p^{s-1}(x)}\right) \cdots\right) \\
& \left.+d_{2} \frac{f(x)}{p(x)}\right)+a_{1} f(x) \\
& =\left(y-y_{1}(x)\right)\left(\left(y-y_{2}(x)\right)\right. \\
& \times\left(\cdots \left(\left(y-y_{s}(x)\right) \lambda \frac{f(x)}{p^{s}(x)}\right.\right. \\
& \left.\left.+d_{s} \frac{f(x)}{p^{s-1}(x)}\right) \cdots\right) \\
& \left.+d_{2} \frac{f(x)}{p(x)}\right)+a_{1} f(x) \text {. }
\end{aligned}
$$

By (55), we have $y_{i}(x)=y_{1}(x)+\lambda_{i} p(x), i=2,3, \ldots, s+$ 1. Then, $F(x, y)$ can be expanded to a power series in the expression:

$$
\begin{aligned}
F(x, y)=( & \left.-y_{1}(x)\right) \\
& \times\left(\left(y-y_{1}(x)-\lambda_{2} p(x)\right)\right. \\
& \times\left(\ldots \left(\left(y-y_{1}(x)-\lambda_{s} p(x)\right) \lambda \frac{f(x)}{p^{s}(x)}\right.\right. \\
& \left.\left.+d_{s} \frac{f(x)}{p^{s-1}(x)}\right) \cdots\right) \\
& \left.+d_{2} \frac{f(x)}{p(x)}\right)+a_{1} f(x) \\
= & \sum_{i=0}^{s} c_{i} \frac{f(x)}{p^{i}(x)}\left(y-y_{1}(x)\right)^{i}
\end{aligned}
$$

for some real numbers $c_{j}, j=0,1, \ldots, s$. Moreover, the leading coefficient of $F(x, y), c_{s}\left(f(x) / p^{s}(x)\right)$ is contained to $\mathbb{R}[x]$, and it follows $p^{s}(x) \mid f(x)$.
Conversely, if $F(x, y)$ is expressed as in Theorem 10, then the cardinal number $\left|\mathrm{Qcs}_{F}\right|=\infty$, this is the same as the sufficient conditions.

Theorem 11. The following two conditions are equivalent:

(i) $F(x, y)=\sum_{i=0}^{s} c_{i}\left(f(x) / p^{i}(x)\right)\left(y-y_{1}(x)\right)^{i}$ for some $y_{1}(x) \in \mathbb{R}[x], p(x)$ is a factor of $f(x)$, and $c_{i} \in \mathbb{R}$ for $i=0,1, \ldots, s$,

(ii) $\left|Q c s_{F}\right|=\infty$. of $\mathbb{R}$.)

(In fact, if $\left|Q \mathrm{cs}_{F}\right|=\infty$, then $\left|\mathrm{Q} \mathrm{cs}_{F}\right|=$ the cardinal number Proof. (i) $\Rightarrow$ (ii) Suppose that (i) holds. Then,

$$
\begin{aligned}
F\left(x, y_{1}(x)+\lambda p(x)\right) & =\sum_{i=0}^{s} c_{i} \frac{f(x)}{p^{i}(x)}(\lambda p(x))^{i} \\
& =\left(\sum_{i=0}^{s} c_{i} \lambda^{i}\right) f(x) \\
& =a p^{m}(x) \quad \text { for } a=\sum_{i=0}^{s} c_{i} \lambda^{i} \in \mathbb{R} .
\end{aligned}
$$

This means that $y_{1}(x)+\lambda p(x) \in \mathrm{Qcs}_{F}$ for each $\lambda \in \mathbb{R}$. It follows that the cardinal $\left|\mathrm{Qcs}_{F}\right|=\infty$.

(ii) $\Rightarrow$ (i) For any $y(x), y_{1}(x) \in \mathrm{Qcs}_{F}$, since $\left|\mathrm{Q} \mathrm{cs}_{F}\right|=\infty$ and by Lemma 7 , we obtain

$$
y(x)-y_{1}(x)=d p(x)
$$

for some factor $p(x)$ of $f(x)$. By Theorem 10, we have

$$
F(x, y)=\sum_{i=0}^{s} c_{i} \frac{f(x)}{p^{i}(x)}\left(y-y_{1}(x)\right)^{i}
$$

for some $y_{1}(x), p(x) \in \mathbb{R}[x]$, and $c_{i} \in \mathbb{R}$ for $i=0,1, \ldots, s$.

If the $F(x, y)$ can be represented as the form of (71) in the following lemma, then any quasi-coincidence solution can be determined.

Lemma 12. Suppose that

$$
F(x, y)=\sum_{i=0}^{s} c_{i} \frac{f(x)}{p^{i}(x)}(y-y(x))^{i},
$$

where $y(x) \in \mathbb{R}[x], c_{i} \in \mathbb{R}, i=0,1, \ldots, s$ and $p(x)$ is a factor of $f(x)$. Then, $h(x) \in \mathbb{R}[x]$ is a quasi-coincidence solution of $F(x, y)$, if and only if

$$
h(x)=y(x)+d p(x) \text { for some } d \in \mathbb{R} .
$$

Proof. At first, we assume that $h(x) \in \mathbb{R}[x]$ is a quasicoincidence solution of $F(x, y)$, and we consider

$$
\begin{aligned}
F(x, y(x)) & =\sum_{i=0}^{s} c_{i} \frac{f(x)}{p^{i}(x)}(y(x)-y(x))^{i} \\
& =c_{0} f(x) .
\end{aligned}
$$

This means $y(x) \in \mathrm{Qcs}_{F}$. 
By Theorem 11,

$$
F(x, y)=\sum_{i=0}^{s} c_{i} \frac{f(x)}{p^{i}(x)}(y-y(x))^{i}, \quad \text { then }\left|\mathrm{Qcs}_{F}\right|=\infty .
$$

It follows from Lemma 7 that for any quasi-coincidence solution $h(x)$, we obtain

$$
h(x)=y(x)+d p(x) \quad \text { for some } d \in \mathbb{R} .
$$

Conversely, suppose $h(x)=y(x)+d p(x)$ for some factor $p(x)$ of $f(x)$ and some constant $d \in \mathbb{R}$. Substituting this $h(x)$ as $y$ in (71), we have

$$
\begin{aligned}
F(x, h(x)) & =F(x, y(x)+d p(x)) \\
& =\sum_{i=0}^{s} c_{i} \frac{f(x)}{p^{i}(x)}(d p(x))^{i} \\
& =\left(\sum_{i=0}^{s} c_{i} d^{i}\right) f(x) .
\end{aligned}
$$

Therefore, $h(x) \in \mathrm{Qcs}_{F}$.

Note that not all polynomial functions $F(x, y)$ can be written as (71). Actually, almost all $F(x, y)$ are expressed as the form of the next theorem. In that situation, any solution can be written as the next form $(\star)$ if the cardinal number $\left|Q s_{F}\right|$ is in nitely many in this theorem.

Theorem 13. Let $F(x, y)$ be a polynomial function with

$$
F(x, y)=a_{s}(x) y^{s}+a_{s-1}(x) y^{s-1}+\cdots+a_{0}(x),
$$

and $f(x)$ a polynomial. If the cardinal number $\left|Q c_{F}\right|$ is infinitely many, then for each quasi-coincidence point solution of (5) must be of the form

$$
-\frac{a_{s-1}(x)}{s a_{s}(x)}+\lambda p(x)
$$

for arbitrary $\lambda \in \mathbb{R}$, where $p(x)=c\left(f(x) / a_{s}(x)\right)^{1 / s}$ is a factor of $f(x)$ and $c$ is a constant.

Proof. Assume $\left|\mathrm{Qcs}{ }_{F}\right|=\infty$. By Theorem 11, we have

$$
\begin{aligned}
F(x, y) & =a_{s}(x) y^{s}+a_{s-1}(x) y^{s-1}+\cdots+a_{0}(x) \\
& =\sum_{i=0}^{s} c_{i} \frac{f(x)}{p^{i}(x)}(y-y(x))^{i}
\end{aligned}
$$

for some $c_{i} \in \mathbb{R}$, and $y(x) \in \mathrm{Qcs}_{F}$. Comparing the coefficients of $y^{s}$ and $y^{s-1}$ in both sides, we get

$$
\begin{gathered}
a_{s}(x)=c_{s} \frac{f(x)}{p^{s}(x)}, \\
a_{s-1}(x)=-s a_{s}(x) y(x)+c_{s-1} \frac{f(x)}{p^{s-1}(x)} .
\end{gathered}
$$

Consequently, by (79) and (80), we get

$$
\begin{gathered}
p^{s}(x)=c_{s} \frac{f(x)}{a_{s}(x)}, \\
y(x)=\frac{c_{s-1}}{s c_{s}} p(x)-\frac{a_{s-1}(x)}{s a_{s}(x)} \in \mathbb{R}[x] .
\end{gathered}
$$

By Lemma 12 and (81), for any $d \in \mathbb{R}$, we have that any quasicoincidence solution is represented by

$$
\begin{aligned}
y(x)+d p(x) & =\frac{c_{s-1}}{s c_{s}} p(x)-\frac{a_{s-1}(x)}{s a_{s}(x)}+d p(x) \\
& =-\frac{a_{s-1}(x)}{s a_{s}(x)}+\left(d-\frac{c_{s-1}}{s c_{s}}\right) p(x) \\
& =-\frac{a_{s-1}(x)}{s a_{s}(x)}+\lambda p(x),
\end{aligned}
$$

where $p(x)=\left(c_{s}\right)^{1 / s}\left(f(x) / a_{s}(x)\right)^{1 / s}$ (note that since $d$ is arbitrary, then $\lambda$ is arbitrary).

This completes the proof.

Finally, we provide two examples. Example 1 explains the case of all cardinal number $\left|\mathrm{Qcs}_{F}\right|=4$.

Example 1. Let

$$
\begin{aligned}
F(x, y)= & \left(x^{2}+x+1\right) y^{2}-x^{6} \\
- & 3 x^{5}-6 x^{4}-7 x^{3}-10 x^{2}-7 x-5, \\
& f(x)=\left(x^{2}+x+1\right)^{2} .
\end{aligned}
$$

Then,

$$
\begin{aligned}
F(x, y)= & \left(x^{2}+x+1\right) \\
& \times\left[y^{2}-\left(x^{2}+x+1\right)^{2}-4\right], \quad \operatorname{deg}_{y} F=s=2 .
\end{aligned}
$$

This polynomial The polynomial equation $F(x, y)=$ $a\left(x^{2}+x+1\right)^{2}$ for some $a \in \mathbb{R}$ has exactly $4=(s+2)$ quasicoincidence solutions as follows:

$$
\begin{gathered}
F\left(x, x^{2}+x+3\right)=4\left(x^{2}+x+1\right)^{2}, \\
F\left(x,-x^{2}-x-3\right)=4\left(x^{2}+x+1\right)^{2}, \\
F\left(x, x^{2}+x-1\right)=-4\left(x^{2}+x+1\right)^{2}, \\
F\left(x,-x^{2}-x+1\right)=-4\left(x^{2}+x+1\right)^{2} .
\end{gathered}
$$

The next example explains that the number of all quasicoincidence solutions of (5) is infinitely many. 
Example 2. Let $x \in \mathbb{R}, f(x)=x^{4}(x-1)^{4}$, and

$$
\begin{aligned}
F(x, y) & =a_{3}(x) y^{3}+a_{2}(x) y^{2}+a_{1}(x) y+a_{0}(x) \\
& =x(x-1) y^{3}+0 y^{2}+x^{3}(x-1)^{3} y+0 .
\end{aligned}
$$

We will solve all quasi-coincidence solutions of $F(x, y)=$ $a x^{4}(x-1)^{4}$ for some $a \in \mathbb{R}$. This polynomial function has at least $6(\geq s+3$, since $s=3)$ quasi-coincidence solutions as follows:

$$
\begin{gathered}
F\left(x_{1}, x_{2}, x^{2}-x\right)=2 x^{4}(x-1)^{4}, \\
F\left(x_{1}, x_{2}, 2 x^{2}-2 x\right)=10 x^{4}(x-1)^{4}, \\
F\left(x_{1}, x_{2},-x^{2}+x\right)=-2 x^{4}(x-1)^{4}, \\
F\left(x_{1}, x_{2},-2 x^{2}+2 x\right)=-10 x^{4}(x-1)^{4}, \\
F\left(x_{1}, x_{2}, \frac{x^{2}}{2}-\frac{x}{2}\right)=\frac{5}{8 x^{4}(x-1)^{4}}, \\
F\left(x_{1}, x_{2},-\frac{x^{2}}{2}+\frac{x}{2}\right)=-\frac{5}{8 x^{4}(x-1)^{4}} .
\end{gathered}
$$

In fact, we have $\left|Q \mathrm{Cs}_{F}\right|=\infty$, and by (79), we obtain

$$
\begin{aligned}
p(x) & =c\left(\frac{f(x)}{a_{s}(x)}\right)^{1 / s} \\
& =c\left(\frac{x^{4}(x-1)^{4}}{x(x-1)}\right)^{1 / 3} \\
& =c x(x-1)
\end{aligned}
$$

for some real number $c$.

By Theorem 13, any quasi-coincidence solution is written as

$$
\begin{aligned}
-\frac{a_{2}(x)}{s a_{3}(x)}+\lambda p(x) & =\frac{0}{3 x(x-1)}+\lambda c x(x-1) \\
& =\mu x(x-1),
\end{aligned}
$$

where $\mu=\lambda c \in \mathbb{R}$ is arbitrary. This shows that the quasicoincidence (point) solutions have cardinal $\left|Q \mathrm{cs}_{F}\right|=\infty$.

We would like to provide one open problem as follows.

Further Development. For a real-valued polynomial function $F: \mathbb{R} \times \mathbb{R} \rightarrow \mathbb{R}$. Can we find all rational quasi-coincidence solutions $y=b(x) / a(x)$ with coprime polynomials $a(x)$, $b(x) \in \mathbb{R}[x]$ to satisfy

$$
F(x, y)=a f(x)
$$

for some polynomials $f(x) \in \mathbb{R}[x]$ ?

\section{Acknowledgments}

The authors wish to express their deep gratitude to Professor Tamaki Tanaka for his valuable comments on this paper and thank the referees for the very useful suggestions and remarks that contributed to the improvement of the paper.

\section{References}

[1] A. K. Lenstra, "Factoring multivariate polynomials over algebraic number fields," SIAM Journal on Computing, vol. 16, no. 3, pp. 591-598, 1987.

[2] S. P. Tung, "Near solutions of polynomial equations," Acta Arithmetica, vol. 123, no. 2, pp. 163-181, 2006.

[3] S. P. Tung, "Algorithms for near solutions to polynomial equations," Journal of Symbolic Computation, vol. 44, no. 10, pp. 1410-1424, 2009.

[4] H.-C. Lai and Y.-C. Chen, "A quasi-fixed polynomial problem for a polynomial function," Journal of Nonlinear and Convex Analysis, vol. 11, no. 1, pp. 101-114, 2010.

[5] Y.-C. Chen and H.-C. Lai, "A non-NP-complete algorithm for a quasi-fixed polynomial problem," Abstract and Applied Analysis, vol. 2013, Article ID 893045, 10 pages, 2013.

[6] R. P. Grimaldi, Discrete and Combinatorial Mathematics, 5/E, 2003. 


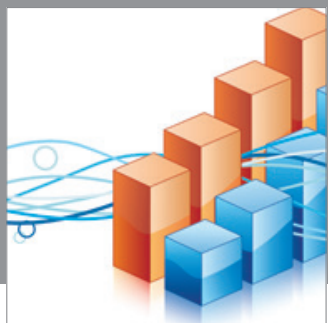

Advances in

Operations Research

mansans



The Scientific World Journal
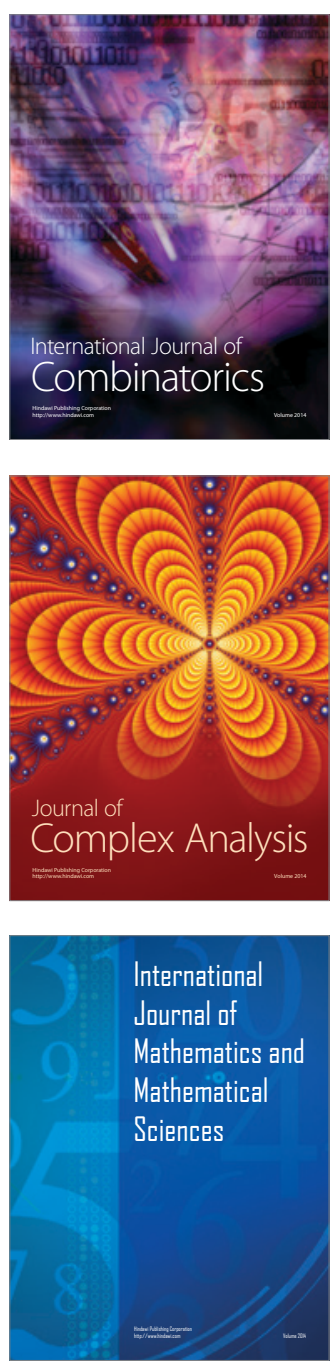


Submit your manuscripts at http://www.hindawi.com


Journal of

Function Spaces



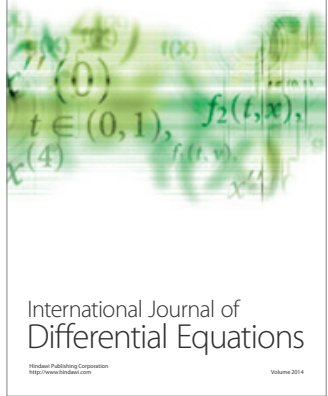
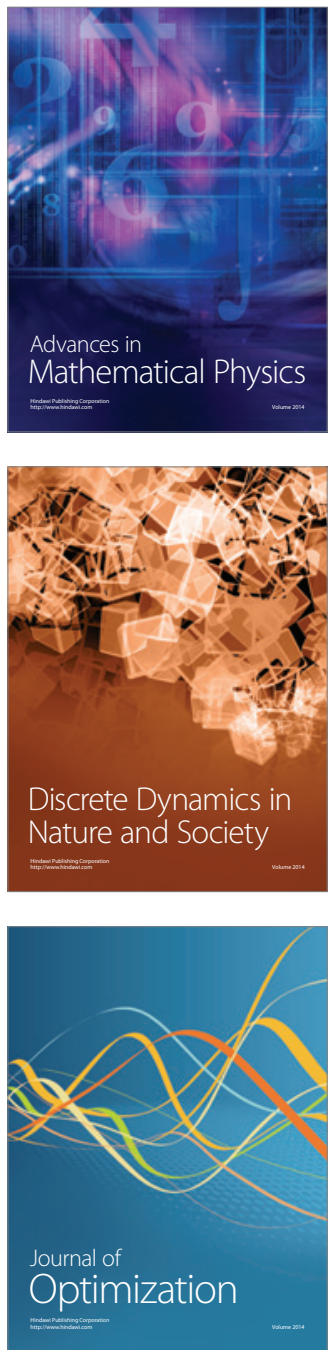